\title{
Front Dynamics during Diffusion-Limited Corrosion of Ramified Electrodeposits
}

\author{
Christophe Léger and Françoise Argoul* \\ Centre de Recherche Paul Pascal, Avenue Schweitzer, 33600 Pessac, France
}

\author{
Martin Z. Bazant \\ Department of Mathematics, Massachusetts Institute of Technology, Cambridge, Massachusetts 02139
}

Received: February 9, 1999; In Final Form: May 1, 1999

\begin{abstract}
Experiments on the diffusion-limited corrosion of porous copper clusters in thin gap cells containing cupric chloride are reported. By carefully comparing corrosion front velocities and concentration profiles obtained by phase-shift interferometry with theoretical predictions, it is demonstrated that this process is well-described by a one-dimensional mean-field model for the generic reaction $\mathrm{A}+\mathrm{B}$ (static) $\rightarrow \mathrm{C}$ (inert) with only one diffusing reactant (cupric chloride) and one static reactant (copper) reacting to produce an inert product (cuprous chloride). The interpretation of the experiments is aided by a mathematical analysis of the model equations, which allows the reaction order and the transference number of the diffusing species to be inferred. Physical arguments are given to explain the surprising relevance of the one-dimensional mean-field model in spite of the complex (fractal) structure of the copper clusters.
\end{abstract}

\section{Introduction}

Diffusion-limited processes are ubiquitous in physics, ${ }^{1}$ chemistry, ${ }^{2}$ and biology. ${ }^{3}$ Reaction-diffusion processes have been the subject of intense and continuous interest since the work of Smoluchowski. ${ }^{4-6}$ A crucial feature of many such processes controlling pattern formation and reaction efficiency is the "reaction front", a dynamic but localized region where reactions are most actively occurring and which separates regions rich in the individual reactants. The simplest theoretical model of a reaction front, introduced more than a decade ago by Gálfi and Rácz, ${ }^{7}$ is the "mean-field" model for two initially separated species A and B reacting to produce an inert species C. Since then, the case of two diffusing reactants $\mathrm{A}$ and $\mathrm{B}$ has been thoroughly studied analytically ${ }^{7-10}$ and numerically, ${ }^{11-21}$ and some predictions of the mean-field model have been checked in the experiments. ${ }^{21-30}$

In contrast, the case of only one diffusing reactant $\mathrm{A}$ and one static reactant $\mathrm{B}$ (confined on a fixed matrix) has not yet been studied experimentally. We show in this paper that the corrosion of a porous solid (B) immersed in a chemically active fluid suspension (A) can also be described by such a meanfield model. Some analytical ${ }^{10,31}$ and numerical ${ }^{11,32}$ studies exist for this case as well, but since it is more microscopically complex (for a real porous interface) than the case of two diffusing reactants (in a homogeneous medium) an experimental test of the model is needed.

The mean-field model of a planar reaction front for the chemical reaction

$$
\mathrm{A}(\text { diffusing })+\mathrm{B} \text { (static) } \rightarrow \mathrm{C} \text { (inert) }
$$

postulates that the concentrations $\rho_{\mathrm{A}}(X, T)$ and $\rho_{\mathrm{B}}(X, T)$ of species $\mathrm{A}$ and $\mathrm{B}$, respectively, evolve according to a pair of coupled partial differential equations ${ }^{10,31}$

$$
\begin{gathered}
\frac{\partial \rho_{\mathrm{A}}}{\partial T}=D_{\mathrm{A}} \frac{\partial^{2} \rho_{\mathrm{A}}}{\partial X^{2}}-R\left(\rho_{\mathrm{A}}, \rho_{\mathrm{B}}\right) \\
\frac{\partial \rho_{\mathrm{B}}}{\partial T}=-R\left(\rho_{\mathrm{A}}, \rho_{\mathrm{B}}\right)
\end{gathered}
$$

where $D_{\mathrm{A}}$ is the diffusion constant for species $\mathrm{A}$ and $R\left(\rho_{\mathrm{A}}, \rho_{\mathrm{B}}\right)$ is the reaction rate density. The most frequently used initial conditions assume that the reactants are uniformly distributed and completely separated at first, $\rho_{\mathrm{A}}(X, 0)=\rho_{\mathrm{A}}^{\circ} H(X)$ and $\rho_{\mathrm{B}}^{\circ}(X, 0)=\rho_{\mathrm{B}}^{\circ} H(-X)$, where $H(X)$ is the Heaviside unit step function. Such initial conditions are easier to reproduce in experiments than those involving uniformly mixed reactants. There are several assumptions behind eqs 2 and 3: (i) The product $\mathrm{C}$ is generated in small enough quantities that its presence does not significantly affect the dynamics. (ii) The concentrations are dilute enough that the diffusivities are constant. (iii) The fixed matrix containing reactant B (static) is porous enough that reactant A can freely diffuse through it. (iv) The reaction rate is a function of only the local concentrations and not any fluctuations or many-body effects (which is the "mean-field approximation"). It is common to make the meanfield approximation under the assumption $R\left(\rho_{\mathrm{A}}, \rho_{\mathrm{B}}\right)=k \rho_{\mathrm{A}}^{m} \rho_{\mathrm{B}}^{n}$, but in the interpretation of our experiments we will not assume anything about the form of $R\left(\rho_{\mathrm{A}}, \rho_{\mathrm{B}}\right)$ a priori since the reaction takes place at a solid-liquid interface. Moreover, this interface is highly ramified, and therefore, the underlying microscopic dynamics is expected to be more complex than for simple homogeneous kinetics.

In this paper we carefully test the validity of these assumptions with experiments on a particular porous-solid corrosion system: copper clusters corroded by a cupric chloride $\left(\mathrm{CuCl}_{2}\right)$ electrolyte. The clusters are obtained by thin gap cell electrodeposition from a $\mathrm{CuCl}_{2}$ electrolyte at fixed current. This process builds a depletion layer of $\mathrm{CuCl}_{2}$ ahead of the copper 


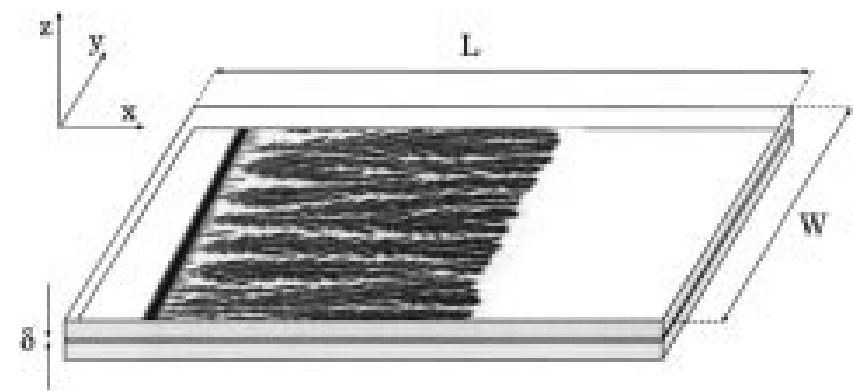

Figure 1. Schematic diagram of the thin gap electrodeposition cell containing a ramified, metallic copper deposit. Note that the size of the deposit has been enlarged for clarity.

deposit. When the current is switched off, the $\mathrm{CuCl}_{2}$ depletion layer relaxes toward the copper cluster, bringing $\mathrm{Cu}^{2+}$ cations that react with copper according to

$$
\mathrm{CuCl}_{2}(\mathrm{aq})+\mathrm{Cu} \text { (solid, red) } \rightarrow 2 \mathrm{CuCl} \text { (solid, white) }
$$

where the cuprous chloride $(\mathrm{CuCl})$ is produced in the form of small (white) crystallites that drop down to the bottom of the cell.

In section II we describe the experimental setup and the method used to prepare the porous clusters to be corroded. In section III we report the experimental evidence that our corrosion system behaves like a $1 \mathrm{D}$ diffusion-reaction process with one static reactant. In section IV, a mathematical analysis is presented that makes quantitative predictions based on the experimental data of section II, within the theoretical framework of the mean-field model, eqs 2 and 3. Also in section IV, the experimental results are revisited to refine the comparison with the theoretical model and to discuss in some detail its physical limitations.

\section{Experimental Methods}

A. Apparatus. The experiments are performed in a thin gap electrodeposition cell, which is depicted schematically in Figure 1. The cell consists of an unsupported, aqueous solution of $\mathrm{CuCl}_{2}$ confined to a narrow region of dimensions $W=5 \mathrm{~cm} \times L=$ $8 \mathrm{~cm} \times \delta=50 \mu \mathrm{m}$ between two closely spaced, optically flat glass plates $(\lambda / 4$ over $80 \mathrm{~mm} \times 50 \mathrm{~mm})$. Two parallel, ultrapure copper and silver wires (50 $\mu \mathrm{m}$ diameter, Goodfellow 99\% purity) are inserted between the two glass plates to act both as spacers and as electrodes. During the electrodeposition (prior to corrosion) the wires are polarized so that the silver wire acts as the cathode and the copper wire as the anode. The solutions of $\mathrm{CuCl}_{2}$ (ACS reagent) are prepared from deionized water, carefully cleaned of any trace of dissolved oxygen by bubbling nitrogen through it for $1 \mathrm{~h}$. The anodic part of the cell (not shown in Figure 1) is filled by a dilute solution of $\mathrm{CuCl}_{2}$ to postpone the precipitation of the salt due to saturation effects by dissolution of the anode. The copper electrodeposits are all grown at constant current, and the entire experiment is performed at room temperature $\left(\approx 20^{\circ} \mathrm{C}\right)$.

Digitized color pictures of the copper clusters are obtained by direct imaging of the cluster through a lens, using a threeCDD camera coupled with an 8-bit frame grabber from Data Translation driven by the public domain software IMAGE, ${ }^{33}$ which successively captures three RGB frames and from them reconstructs the color image.

A phase-shift Mach Zehnder interferometer is used independently to resolve the concentration field, averaged over the depth of the cell. A sketch of the interferometer can be found in ref
34. The interference patterns are recorded through a CCD camera coupled to the same frame grabber ${ }^{33}$ with a $768 \times 512$ pixel resolution. Phase-shift interferometry offers several significant advantages over traditional interferometry in that it provides an accurate reconstruction of the entire concentration field, using a set of successive interference pictures recorded for shifted values of the phase difference between two optical wavefronts and can also be used as an holographic interferometer. ${ }^{35}$

B. Preparation of Copper Clusters by Electrodeposition. When current flows from the anode to the cathode, charge transfer occurs at the cathode, leading to the reduction of copper cations into copper metal according to ${ }^{34,36,37}$

$$
\mathrm{Cu}^{2+}+2 \mathrm{e}^{-} \rightarrow \mathrm{Cu}
$$

The actual mechanism of deposition is much more complex than this two-electron transfer process since competitive reactions involving the solvent species are likely to occur. Nevertheless, in $\mathrm{CuCl}_{2}$ electrolytes, we have observed that the formation of cuprous oxide $\left(\mathrm{Cu}_{2} \mathrm{O}\right)$ in competition with copper by reduction of $\mathrm{Cu}^{2+}$ cations is not favored, contrary to what is observed in copper sulfate $\left(\mathrm{CuSO}_{4}\right)$ solutions, ${ }^{38-40}$ which can be partly explained by the strong adsorption and complexation properties of chloride anions. ${ }^{37}$ This reduction process on the cathode implies a local depletion of the copper cations close to the cathode and also their replenishment by a global transport process, namely diffusion. Although electromigration also contributes to transport, it does not act independently of diffusion in regions where electroneutrality is maintained, ${ }^{41}$ which means everywhere in the cell outside the $10-100 \AA$ thick double layer. ${ }^{42,43}$ This often misunderstood fact was given a firm theoretical basis by Newman over 30 years ago in his asymptotic analysis of the transport equations for a rotating disk electrode, ${ }^{44}$ but only recently has it been quantitatively verified in experiments (by our group) for the case of constant boundary flux at a fixed cathode. ${ }^{34,36}$ In summary, the theoretical and experimental evidences indicate that in the absence of convection the concentration $\rho_{\mathrm{A}}$ of a dilute, binary electrolyte evolves according to the classical diffusion equation

$$
\frac{\partial \rho_{\mathrm{A}}}{\partial t}=D_{\mathrm{A}} \nabla^{2} \rho_{\mathrm{A}}
$$

where $D_{\mathrm{A}}$ is the "ambipolar diffusion coefficient" for the electrolyte given by a certain weighted average of the diffusion constants of the individual ions. ${ }^{41}$

When the interfacial concentration of metal cation $\mathrm{Cu}^{2+}$ approaches zero, the interface becomes unstable and develops into a forest of fine spikes that compete with each other to invade the cell. ${ }^{45,46}$ In some cases, a "dense-branched" pattern is selected. ${ }^{36,47-50}$ This morphology is characterized by a dense array of branches of invariant width advancing at constant velocity $v$ through the cell, whose tips delimit a nearly planar front between the copper salt electrolyte and the deposit zone. We have shown recently that this growth regime can be modeled via a $1 \mathrm{D}$ diffusion model through the measurement of the copper salt concentration field ahead of the growing deposit by interferometry. ${ }^{36,50}$ The experimental concentration field closely fits the "traveling-wave" solution to eq 5 ,

$$
\rho_{\mathrm{A}}(X)=\rho_{\mathrm{A}}^{\circ}\left(1-\exp ^{-X / L_{\mathrm{d}}}\right)
$$

where $L_{\mathrm{d}}=D_{\mathrm{A}} / v$, and $X$ is the distance to the front edge of the copper deposit, in the direction normal to the front, oriented 
toward the bulk electrolyte. The diffusion length $L_{\mathrm{d}}$ is proportional to $\rho_{\mathrm{A}}^{\circ} / j$, where $\rho_{\mathrm{A}}^{\circ}$ is the initial bulk concentration in copper cations and $j$ is the current density. This diffusion length tends to zero as $j / \rho_{\mathrm{A}}^{\circ}$ increases, and in that limit the concentration profile looks like a step function. Note that $\rho_{\mathrm{A}}(X \leq 0) \approx 0$ and $\rho_{A}\left(X \gg L_{\mathrm{d}}\right) \approx \rho_{\mathrm{A}}^{\circ}$; i.e., the metallic copper deposit leaves behind it a region entirely depleted in copper cations, pushing in front of it a diffusion layer of constant width extending into the bulk electrolyte. Due to the conservation of copper during the deposition process, a linear relation exists between the velocity $v$ of the growth and the interfacial flux of cations $J$, namely $v \rho_{\mathrm{B}}=J$, where $\rho_{\mathrm{B}}$ is the mean concentration of (metallic) copper in the region of the deposit.

Using the relation $v=D_{\mathrm{A}} / L_{\mathrm{d}}$, the ratio of the copper concentration in the bulk electrolyte $\rho_{\mathrm{A}}^{\circ}$ (where it takes the form of cupric ions) to that in the region of the deposit $\rho_{\mathrm{B}}^{\circ}$ (where it mostly takes the metallic form) is easily calculated from the basic properties of the electrolyte $36,48-50$

$$
q \equiv \frac{\rho_{\mathrm{A}}^{\circ}}{\rho_{\mathrm{B}}^{\circ}}=1-t^{+}
$$

where $t^{+}$is the transference number ${ }^{41,43}$ of the copper cation in a $\mathrm{CuCl}_{2}$ electrolyte. Practically, $t^{+}$is a characteristic of the electrolyte and therefore $q$ will not be a free parameter in our experiments (neither $t^{+}$nor $q$ depend on the current density $j$ ). The closer $t^{+}$is to 1 , the greater the concentration of copper inside the cluster. In $\mathrm{CuCl}_{2}$ electrolytes, $t^{+}$is expected to be smaller than 0.5 , which implies that the copper composition of the deposited zone will not go beyond twice the original concentration of $\mathrm{CuCl}_{2}$ in the electrolyte. Therefore, the copper clusters obtained by thin gap electrodeposition in $\mathrm{CuCl}_{2}$ are in fact highly porous.

The large porosity of the deposited copper clusters is of fundamental importance in our subsequent study of the corrosion of the copper deposits once the current has been switched off (and the electrodeposition halted) because, as a consequence, the cupric ions are able to diffuse freely through the dendrites with approximately their bulk diffusivity and then react with a large exposed surface of metallic copper. The low density of the deposit also suggests that the product of the corrosion reaction, cuprous chloride $(\mathrm{CuCl})$ crystal, is produced in small enough quantities that its presence should not significantly affect the dynamics of the reaction-diffusion process. Therefore, by interrupting the current during electrodeposition, we can observe a simple reaction-diffusion system with two initially separated reactants, copper chloride (A) and metallic copper (B), only one of which is free to diffuse. Since the initial interface between the bulk electrolyte and the ramified electrodeposit is planar and the deposit is disordered, it is likely that the dynamics of the corrosion process will be effectively "one-dimensional" (1D), in the sense that there might be nearly perfect translational symmetry in the two spatial directions ( $Y$ and $Z$ ) perpendicular to direction of the front propagation. Moreover, since the dynamics occurs in three dimensions (as opposed to two for a surface or one for a molecular channel), it is also likely that a mean-field, continuum model will be valid, although this may not seem obvious a priori in light of the complex geometry of the electrodeposits, which is known to be fractal. ${ }^{51-54}$

The rest of the paper is devoted to a careful, experimental validation of these hypotheses, showing that our system is indeed accurately described by a one-dimensional, mean-field model for the generic chemical reaction, $\mathrm{A}+\mathrm{B}$ (static) $\rightarrow \mathrm{C}$. We begin

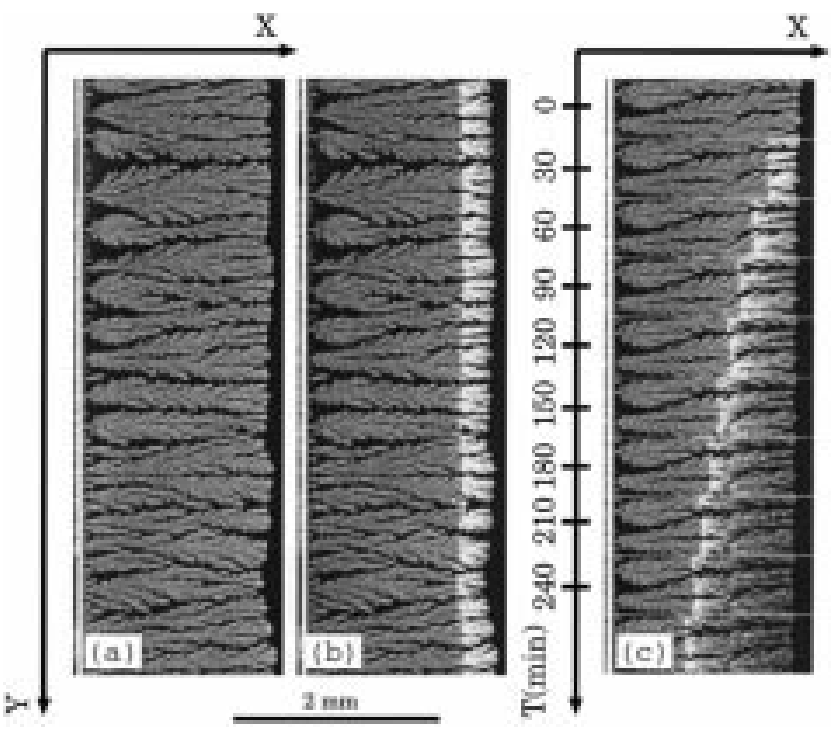

Figure 2. (a) Photograph of a copper deposit grown from a $0.5 \mathrm{~mol} \cdot \mathrm{L}^{-1}$ $\mathrm{CuCl}_{2}$ solution at $j=40 \mathrm{~mA} \cdot \mathrm{cm}^{-2}$ for approximately $15 \mathrm{~min}$. (b) Photograph of the same deposit $0.5 \mathrm{~h}$ after the current had been switched off. (The white zone is $\mathrm{CuCl}$.) (c) The montage shows a sequence of photographs of a small region of the deposit including the reaction front taken every $30 \mathrm{~min}$ after the interruption of the current.

in the next section by describing the scaling behavior of the reaction front and accompanying depletion layer of $\mathrm{CuCl}_{2}$. In the following section, a mathematical analysis of the onedimensional, mean-field model is presented, which incorporates the observed scalings and makes quantitative predictions regarding the reaction front speed and the concentration evolution. Finally, these predictions are checked with a more detailed analysis of the experimental data in the last section, and arguments are given to explain the relevance of the onedimensional, mean-field model for our experimental system.

\section{Preliminary Experimental Results}

A. Temporal Evolution of the Corrosion Front. At the moment when the current is switched off, the region of the copper deposit is entirely depleted of cupric ions, which are thus initially separated from the metallic copper in the deposit. At later times, cupric ions diffuse amidst the copper dendrites and react at the metal surfaces, leaving behind cuprous chloride $(\mathrm{CuCl})$ crystallites. In Figure 2a,b are shown images of a copper deposit just prior to corrosion and after $30 \mathrm{~min}$ of corrosion, respectively. Note that in Figure $2 b$ the interfacial region between the red copper (the gray color in this picture) and the white $\mathrm{CuCl}$ is rather flat and thin.

Focusing on the temporal evolution of this red/white interface, we have observed that, while at first the white layer of $\mathrm{CuCl}$ appears at the tips of the copper-deposit branches, it gradually becomes flatter and flatter. As a result, the system approaches translational invariance along the $Y$ direction, normal to the growth direction $X$, thus justifying a one-dimensional model for the system involving the single spatial coordinate $X$ (normal to the reaction front).

By carefully comparing the concentration field of cupric ions obtained by phase-shift interferometry and the red/white, $\mathrm{Cu}$ / $\mathrm{CuCl}$ interface observed on the deposit, the location and extent of the reaction front, where there is a significant overlap of metallic copper and cupric ions, can be identified. Following a transient regime (which we describe in the last section), it is observed that the reaction front approaches a constant width $w$ 


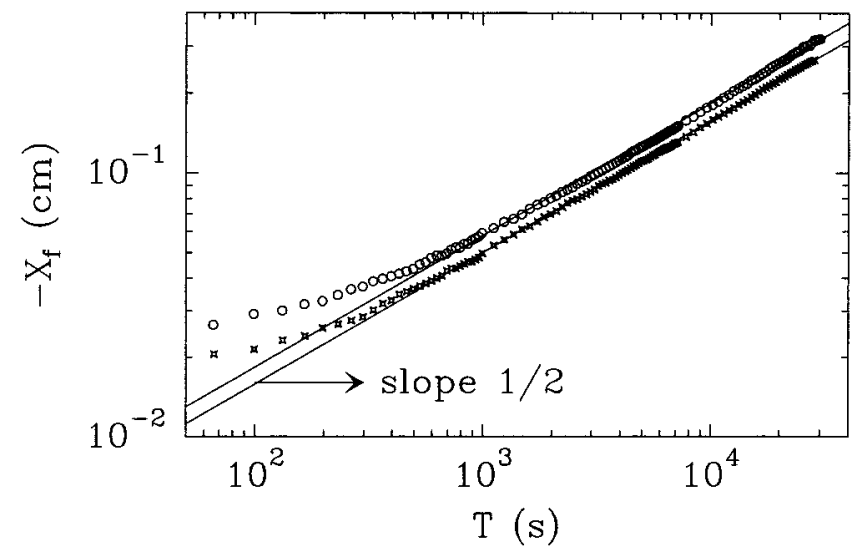

Figure 3. $\log -\log$ plot of the position of the reaction front $X_{\mathrm{f}}$ as a function of time $T$ for two different experimental runs in $\mathrm{CuCl}_{2} 0.5$ $\mathrm{mol} \cdot \mathrm{L}^{-1}$ for deposits grown at $j=40 \mathrm{~mA} \cdot \mathrm{cm}^{-2}$. The solid lines of slope $1 / 2$ represent the predictions of the one-dimensional, mean-field theory, given by eq 41 , with $D=10^{-5} \mathrm{~cm}^{2} \cdot \mathrm{s}^{-1}$, in the cases $q=0.6$ and $q=0.73$.

$\sim T^{\alpha}$ with $\alpha=0$, which is consistent with certain mean-field models. ${ }^{10,11,31}$ Using the theoretical methods pioneered by Gálfi and Rácz $^{7}$ in the case of two diffusing reactants, this scaling was first predicted by Jiang and Ebner ${ }^{11}$ using physical arguments supported by computer simulations and later by $\mathrm{Koza}^{31}$ using asymptotic analysis.

Recently, Bazant and Stone ${ }^{10}$ have considered the case of higher-order reactions $m \mathrm{~A}+n \mathrm{~B}$ (static) $\rightarrow \mathrm{C}$ represented by the mean-field reaction rate $R\left(\rho_{\mathrm{A}}, \rho_{\mathrm{B}}\right)=k \rho_{\mathrm{A}}^{m} \rho_{\mathrm{B}}^{n}$ and proved that the scaling exponent for the front width is given (uniquely) by the formula

$$
\alpha=\frac{m-1}{2(m+1)}
$$

which holds for any real number $m \geq 1$. (The scaling solution does not exist for $m<1$.) In light of this result, the experimental observation $\alpha=0$ is consistent with the usual one-dimensional, mean-field theory only in the case $m=1$. If higher-order reactions were present, $m>1$, the theory would predict that the reaction front width increases in time $(\alpha>0)$ although always more slowly than diffusion $(\alpha<1 / 2)$.

The position of the reaction front $X_{\mathrm{f}}(T)$ during the corrosion of a copper deposit (grown from a $0.5 \mathrm{M} \mathrm{CuCl}_{2}$ solution at $j=$ $40 \mathrm{~mA} / \mathrm{cm}^{2}$ ) is plotted in Figure 3. Note that, after initial transients have vanished $(T>500 \mathrm{~s})$, the reaction front itself "diffuses" with its position given by the scaling, $X_{\mathrm{f}} \sim T^{\sigma}$ with $\sigma=1 / 2$, which is is also consistent with predictions of the onedimensional mean-field model. ${ }^{7,11,31}$

B. Temporal Evolution of the Diffusion Layer. At $T=0$ when the current is interrupted, the reactants $\mathrm{Cu}$ and $\mathrm{CuCl}_{2}$ are completely separated, since the concentration of $\mathrm{CuCl}_{2}$ is negligibly small in the immediate vicinity of the metallic $\mathrm{Cu}$ electrodeposit. During the subsequent corrosion process the concentration of $\mathrm{CuCl}_{2}$ remains very small in the reaction front, which leads to the modification of the initial depletion layer of $\mathrm{CuCl}_{2}$ (produced by the electrodeposition process) into a region where the concentration smoothly interpolates to the value of the bulk solution far behind the front. The term "diffusion layer" is used to describe this region because it is characterized by the transport of fresh $\mathrm{CuCl}_{2}$ by diffusion from the bulk, relatively unaffected by chemical reactions due to the negligible (or vanishing) concentration of metallic $\mathrm{Cu}$ remaining behind the reaction front.
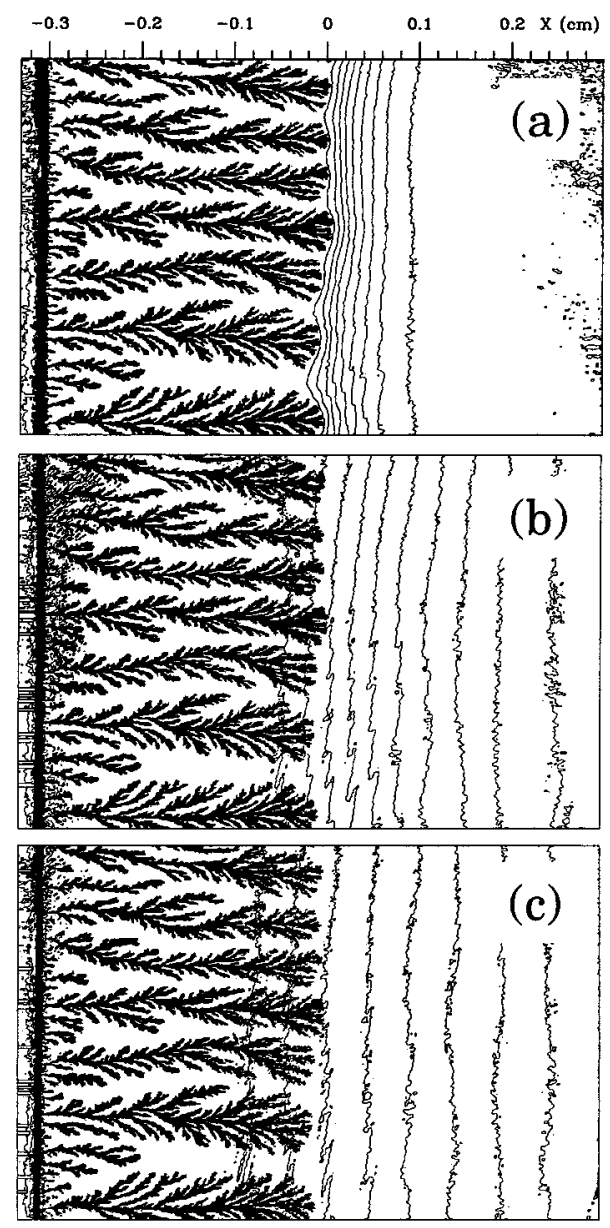

Figure 4. Interferometric characterization of the concentration field around a copper deposit during its dissolution (a) just before the interruption of the current, (b) 15 min later, and (c) $1 \mathrm{~h}$ later. $(\Delta \rho \approx$ $\rho_{\mathrm{A}}^{\circ} / 10$ between adjacent isoconcentration lines.) The deposit grown in $0.5 \mathrm{~mol} \cdot \mathrm{L}^{-1} \mathrm{CuCl}_{2}$ solution at $j=40 \mathrm{~mA} \cdot \mathrm{cm}^{-2}$ for $20 \mathrm{~min}$. The concentration of $\mathrm{CuCl}_{2}$ is negligibly small inside and ahead (to the left) of the reaction front and approaches the bulk value of $0.5 \mathrm{~mol} \cdot \mathrm{L}^{-1}$ far behind (to the right of) the front.

The temporal evolution of the diffusion layer is revealed by precise interferometric measurements of the concentration profile of $\mathrm{CuCl}_{2}$. In Figure 4 are shown three contour plots of the concentration field computed from the integrated index along the depth of the cell. Since the experiments are performed in thin gap cells $(50 \mu \mathrm{m})$ and the depletion layer spreads over distances larger than this gap, it is safely assumed that the concentration of $\mathrm{CuCl}_{2}$ does not change appreciably along the $Z$ direction (parallel to the laser beam ${ }^{36}$ ). In Figure 4, the shadow of the $\mathrm{Cu} / \mathrm{CuCl}$ cluster is also clearly seen. A close inspection of the panels (b) and (c), which correspond to eroded clusters, reveals that in the zone of the copper cluster where $\mathrm{CuCl}_{2}$ has diffused (recognizable where the leftmost isoconcentration contours have moved through the cluster), the cluster has been broken down into smaller crystallites, which, as indicated by their color in Figure 2, are made of $\mathrm{CuCl}$.

Typical experimental concentration profiles of $\mathrm{CuCl}_{2}$ measured at different times (averaged along the $Y$ direction, normal to the growth direction) are shown in Figure 5. The shape of these concentration profiles is discussed in the next two sections, but here we focus on the scaling of the width $W_{\mathrm{d}}(T)$ of the diffusion layer (defined as the region of non-negligible gradients). Figure 6 shows that at long times $(T>500 \mathrm{~s})$, the diffusion layer approaches a self-similar structure, with the diffusive flux entering the reaction front obeying the scaling 


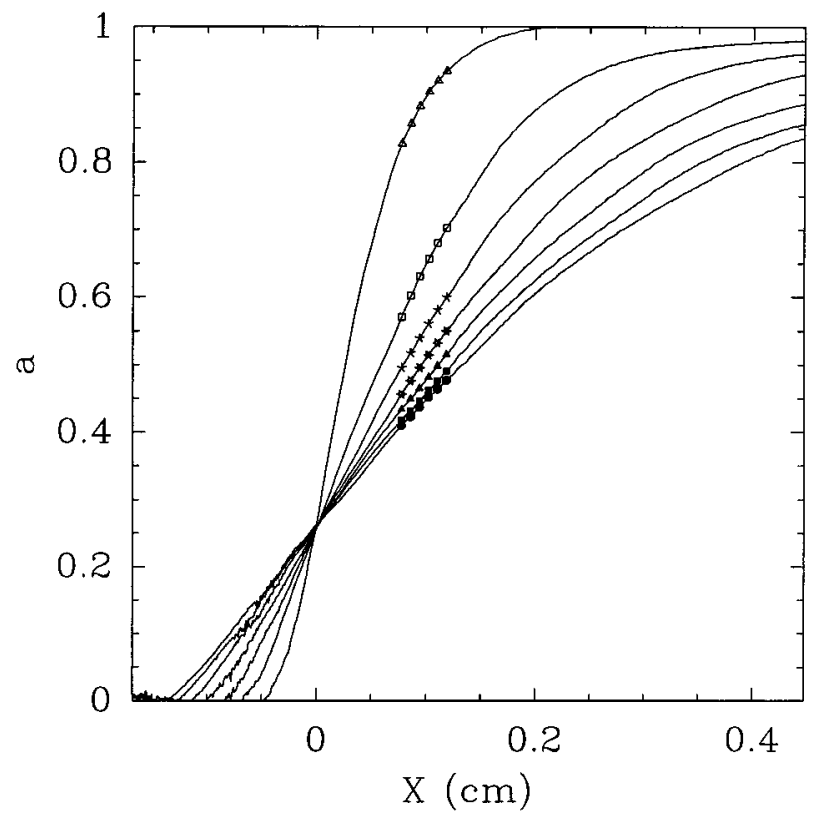

Figure 5. One-dimensional concentration profiles extracted from the two-dimensional data. The deposit has been grown from a $1 \mathrm{~mol} \cdot \mathrm{L}^{-1}$ $\mathrm{CuCl}_{2}$ solution at $j=68 \mathrm{~mA} \cdot \mathrm{cm}^{-2}$ over $15 \mathrm{~min}$. The concentration profiles are shown every $15 \mathrm{~min}$ after the current had been switched off. The different symbols are added on each profile to differentiate the recording times. These symbols will be used on the next representations of the concentration profiles in Figures 9 and 10 .

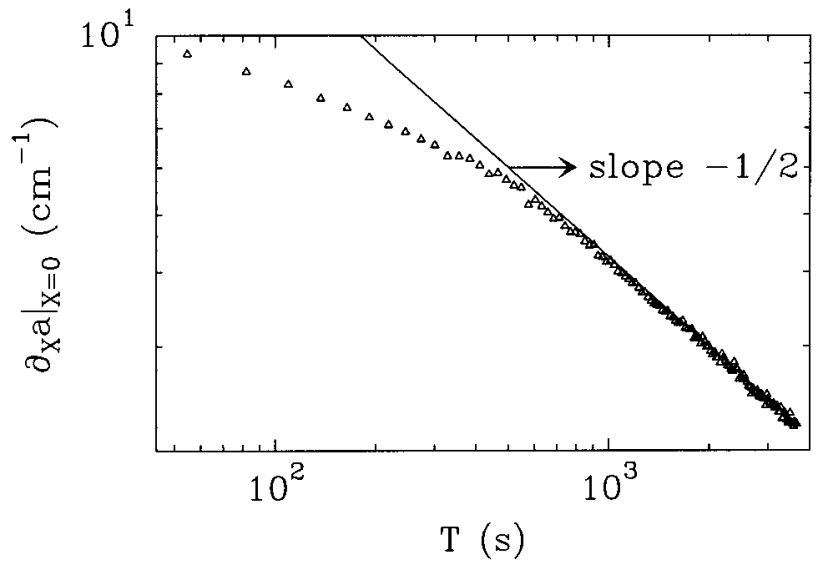

Figure 6. $\log -\log$ plot of the temporal evolution of the derivative of $a(X, T)$ at $X=0$ as a function of $T$. Same parameters as in Figure 5. The plain line corresponds to the prediction of eq 45 with $D=10^{-5}$ $\mathrm{cm}^{2} \cdot \mathrm{s}^{-1}$ and $q=0.79$.

law $\left.J_{\mathrm{d}} \propto\left(\partial \rho_{\mathrm{A}} / \partial X\right)\right|_{X=X_{\mathrm{f}}} \sim T^{-\delta}$, and that, therefore, the width of the diffusion layer has the familiar scaling ${ }^{7,9,11,31} W_{\mathrm{d}} \sim T^{\delta}$ with $\delta=1 / 2$, which is another robust feature of the mean-field models. ${ }^{10}$

A physical argument based on mass conservation between the diffusion layer and reaction front ${ }^{7,11}$ can be used to predict the scaling of the reaction rate (per unit volume) in the front $R$ $\sim T^{-\beta}$ from the preceding experimental observations. The total reaction rate in the front (per unit area) scales as $w R \sim T^{\alpha-\beta}$, and this flux of cupric ions due to reactions must balance the diffusive flux entering the front $J_{\mathrm{d}} \sim T^{-\delta}$, which yields the scaling relation, $\beta=\alpha+\delta=0+1 / 2=1 / 2$. It is important to point out, however, that while $\alpha=0$ and $\delta=1 / 2$ are the results of direct experimental observations, the scaling exponent $\beta=$ $1 / 2$ is only inferred by a physical argument, based on the assumption that chemical reactions are negligible in the diffusion layer. Although this assumption has been checked numerically and analytically for various mean-field models, the reaction rate is not directly measured in our experiments.

In the general case $R\left(\rho_{\mathrm{A}}, \rho_{\mathrm{B}}\right)=k \rho_{\mathrm{A}}^{m} \rho_{\mathrm{B}}^{n}$ mentioned above, it can be shown ${ }^{10}$ that $\beta$ is given (uniquely) by

$$
\beta=\frac{m}{m+1}
$$

so that once again $m=1$ is suggested by the inferred value $\beta$ $=1 / 2$. However, given that the experimental system has a complex fractal structure and three-dimensional transport in the reaction front, it is not obvious a priori that $R\left(\rho_{\mathrm{A}}, \rho_{\mathrm{B}}\right)=k \rho_{\mathrm{A}}^{m} \rho_{\mathrm{B}}^{n}$ is a reasonable approximation within a spatially averaged onedimensional model. Instead, we will make no ad hoc assumptions about the functional form of the reaction rate $R\left(\rho_{\mathrm{A}}, \rho_{\mathrm{B}}\right)$ and then explore consequences of only our direct experimental observations, $\alpha=0$ and $\delta=\sigma=1 / 2$, within the framework of a one-dimensional mean-field model.

\section{Theoretical Predictions of the Mean-Field Model}

A. Dimensionless Model Equations. The model equations have a dimensionless form involving only the parameter, $q \equiv$ $\rho_{\mathrm{A}}^{\circ} / \rho_{\mathrm{B}}^{\circ}$, defined in eq 7 ,

$$
\begin{gathered}
\frac{\partial a}{\partial t}=\frac{\partial^{2} a}{\partial x^{2}}-r(a, b) \\
\frac{\partial b}{\partial t}=-q r(a, b)
\end{gathered}
$$

with boundary and initial conditions

$$
\begin{array}{cc}
a(-\infty, t)=0, & a(\infty, t)=1 \\
b(-\infty, t)=1, & b(\infty, t)=0 \\
a(x, 0)=H(x), & b(x, 0)=H(-x)
\end{array}
$$

where

$$
\begin{gathered}
a \equiv \frac{\rho_{\mathrm{A}}}{\rho_{\mathrm{A}}^{\circ}}, \quad b \equiv \frac{\rho_{\mathrm{B}}}{\rho_{\mathrm{B}}^{\circ}} \\
r(a, b) \equiv \frac{R\left(a \rho_{\mathrm{A}}^{\circ}, b \rho_{\mathrm{B}}^{\circ}\right)}{R\left(\rho_{\mathrm{A}}^{\circ}, \rho_{\mathrm{B}}^{\circ}\right)} \\
t \equiv \frac{R\left(\rho_{\mathrm{A}}^{\circ}, \rho_{\mathrm{B}}^{\circ}\right) T}{\rho_{\mathrm{A}}^{\circ}}, \quad x \equiv X \sqrt{\frac{R\left(\rho_{\mathrm{A}}^{\circ}, \rho_{\mathrm{B}}^{\circ}\right)}{\left(D_{\mathrm{A}} \rho_{\mathrm{A}}^{\circ}\right)}}
\end{gathered}
$$

These initial conditions are closest to the actual ones used in the experiments when the copper deposit is grown at large current, which corresponds to small $L_{\mathrm{d}}$ in eq 6 . The initialboundary-value problem of eqs 10-14 involves an idealized, infinite system possessing no natural length or time scale, and therefore, it is expected that asymptotic similarity solutions exist in which distance and time appear coupled by power-law scalings. ${ }^{55}$ The experimental system, on the other hand, possesses several relevant length scales, but they turn out not to affect the evolution of the reaction front, at least for some range of times. For example, the spatial scales of the copper deposit, such as the typical dendrite spacing and dendrite width, surely affect the dynamics at early times since these length scales are of the same order as the diffusion length $L_{\mathrm{d}},{ }^{36}$ but it is observed that during corrosion the system quickly approaches planar symmetry, averaged across scales much larger than individual dendrites. Likewise, the length scale of the gap spacing is not 
expected to greatly influence the corrosion dynamics because vertical (buoyancy-driven) convection, which has been observed during the growth phase ${ }^{56}$ is suppressed in $50 \mu \mathrm{m}$ depth cells. ${ }^{34,57}$ However, the settling of the reaction product, $\mathrm{CuCl}$ crystallites, could have some effect on the front dynamics at this scale. Finally, the largest length scales, namely the distances from the outer edge of the deposit to the two electrodes, also should not affect corrosion dynamics until the reaction front gets close to the cathode and/or the diffusion layer approaches the anode. Therefore, during intermediate times, after three-dimensional transient effects have subsided but before the system size begins to matter, the corrosion dynamics should be well described by a self-similar solution to the one-dimensional mean-field equations.

B. The Diffusion Layer. Motivated by these arguments, we consider the transformation

$$
a(x, t)=\tilde{A}(\xi, t), \quad b(x, t)=\tilde{B}(\xi, t), \quad \xi=\frac{x-x_{\mathrm{f}}(t)}{W_{\mathrm{d}}(t)}
$$

for the concentration of $\mathrm{CuCl}_{2}$ in the diffusion layer (defined by $\zeta>0$ ) with power-law expressions for $x_{\mathrm{f}}(t)$ and $W_{\mathrm{d}}(t)$. In light of the experimental results from the previous section we make the definitions $W_{\mathrm{d}}(t)=2 \sqrt{t}$ and

$$
\begin{gathered}
x_{\mathrm{f}}(t)=-2 v \sqrt{t} \\
\zeta=\frac{x}{2 \sqrt{t}}+v
\end{gathered}
$$

where $v(q)^{2}$ is an effective diffusion constant for the reaction front to be determined during the analysis. Substituting these expressions into eq 10 , we have

$$
\frac{\partial \tilde{A}}{\partial t}+\left(\frac{v-\zeta}{2 t}\right) \frac{\partial \tilde{A}}{\partial \zeta}=\left(\frac{1}{4 t}\right) \frac{\partial^{2} \tilde{A}}{\partial \xi^{2}}-r(\tilde{A}, \tilde{B})
$$

which simply amounts to a change of variables from $(x, t)$ to $(\xi, t)$.

The starting point for our analysis is the experimental observation (see below) that the concentration of $\mathrm{CuCl}_{2}$ approaches a single, continuous profile in the $\zeta$ coordinate

$$
\lim _{t \rightarrow \infty} \tilde{A}(\zeta, t)=A(\zeta) \quad(\zeta>0)
$$

which has been called the "quasistationary approximation" in the physics literature. ${ }^{9,15,31,58}$ This is not really an "approximation" but is rather an exact asymptotic property of a certain class of solutions to eqs $10-13$, which happens to fit the experimental data. To find such solutions from eq 21 , we must assume $t(\partial \tilde{A} /$ $\partial t) \rightarrow 0, \partial \tilde{A} / \partial \xi \rightarrow A^{\prime}(\xi)$, and $\partial^{2} \tilde{A} / \partial \xi^{2} \rightarrow A^{\prime \prime}(\xi)$ for fixed $\zeta>0$. At this point it is customary ${ }^{31}$ to further assume ad hoc that the reaction term in eq 21 vanishes relative to the diffusion term

$$
\lim _{t \rightarrow \infty} t \cdot r(\tilde{A}, \tilde{B})=0 \quad(\zeta>0)
$$

and that the concentration of the nondiffusing species also vanishes in the diffusion layer, i.e., where the reaction front has already passed,

$$
\lim _{t \rightarrow \infty} \tilde{B}(\zeta, t)=0 \quad(\zeta>0)
$$

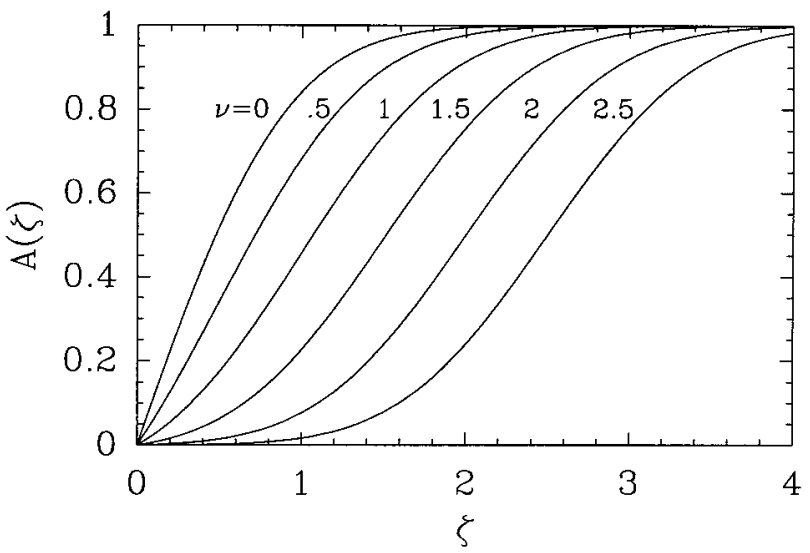

Figure 7. Asymptotic similarity function $a(x, t) \sim A(\xi)$, where $\zeta=$ $(x / 2 \sqrt{t})+v$, shown for $A_{\mathrm{o}}=0$ and $v=0,0.5,1,1.5,2,2.5$ from left to right.

but Bazant and Stone have shown that these limits are actually necessary consequences of the assumed quasistationarity. ${ }^{10}$

With these arguments we are led to an ordinary differential equation for $A(\xi)$ by taking the limit $t \rightarrow \infty$ with fixed $\zeta>0$ in eq 21:

$$
A^{\prime \prime}+2(\zeta-v) A^{\prime}=0
$$

The solution of this equation subject to the boundary condition $A(\infty)=1$ can be written in terms of error functions, ${ }^{59}$

$$
A(\zeta)=A_{\mathrm{o}}+\left(1-A_{\mathrm{o}}\right) \frac{\operatorname{erf}(\zeta-v)+\operatorname{erf}(v)}{1+\operatorname{erf}(v)}
$$

where $A_{\mathrm{o}} \equiv A(0)$ is a constant to be determined by asymptotic matching with the reaction front as $\zeta \rightarrow 0$. The function $A(\zeta)$ is shown in Figure 7 for different values of $\nu$. The slope of $A(\xi)$ at $\zeta=0$ given by

$$
A^{\prime}(0)=\frac{2\left(1-A_{\mathrm{o}}\right) \mathrm{e}^{-v^{2}}}{\sqrt{\pi}(1+\operatorname{erf}(v))}
$$

is the (dimensionless) diffusive flux into the reaction front.

On the length scale $W_{\mathrm{d}}(t) \propto t^{1 / 2}$ appropriate for the diffusion layer, the limiting concentration fields just derived appear not to be differentiable at $\zeta=0$,

$$
a(x, t) \sim A(\xi) H(\xi), \quad b(x, t) \sim H(-\zeta)
$$

as $t \rightarrow \infty$ with $\zeta \neq 0$ fixed, but as we have already observed experimentally, that is only because in the reaction front (at $\zeta=0$ ) the concentrations are smoothly interpolated across these apparent discontinuities on a much smaller length scale $w \propto t^{\alpha}=o\left(W_{\mathrm{d}}\right)$ since $\alpha<\delta$. In mathematical terms, the asymptotic approximations in eq 28 are not uniformly valid for all $(x, t)$ as $t \rightarrow \infty$, but rather are valid only for $\xi \neq 0$, i.e. $\sqrt{t}=$ $O(|x+2 v \sqrt{t}|)$.

C. The Reaction Front. We now explore the consequences of the experimental results $\alpha=0$ and $\delta=\sigma=1 / 2$ within the present mathematical model. Although the physical arguments made above for the lack of a natural length scale are much more tenuous in the reaction front because the observed front width (about $0.2 \mathrm{~mm}$ ) is comparable to the average dendrite thickness $(0.1 \mathrm{~mm})$ and spacing $(0.4 \mathrm{~mm})$ as well as the gap $(0.05 \mathrm{~mm})$, the nearly perfect planar symmetry of the corrosion process leads us to nevertheless seek another asymptotic similarity solution to the one-dimensional, mean-field equations in the vicinity of 
the reaction front, $x-x_{\mathrm{f}}(t)=O(1)$. The predictions of the model will be carefully tested against the experimental data in the next section.

Since $\alpha=0$ and $\sigma=1 / 2$, we consider the transformation

$$
a(x, t)=t^{-\gamma} \mathrm{A}^{\tilde{\tau}}(\eta, t), \quad b(x, t)=\mathrm{B}^{\tilde{}}(\eta, t)
$$

where $\eta$ is a new similarity variable for the reaction front defined by

$$
\eta=x+2 v t^{1 / 2}=2 t^{1 / 2} \xi
$$

The exponent $\gamma \geq 0$ is introduced to allow for the possibility that $a(x, t) \rightarrow 0$ in the reaction front, which is suggested by the result $r(a, b) \sim t^{-\beta}$ with $\beta=1 / 2$ inferred earlier from the experimental data. In contrast, no such prefactor multiplies $\mathrm{B}^{\sim}(\eta, t)$ in the reaction front since $b(x, t)$ must remain finite there in order to interpolate between the limiting values of 0 and 1 , respectively, behind and ahead of the front.

Making these transformations in eq 10 yields

$$
\frac{\partial \mathrm{A}^{\sim}}{\partial t}+v t^{-1 / 2} \frac{\partial \mathrm{A}^{\sim}}{\partial \eta}-\gamma t^{-1} \mathrm{~A}^{\sim}=\frac{\partial^{2} \mathrm{~A}^{\sim}}{\partial \eta^{2}}-t^{\gamma} r\left(t^{-\gamma} \mathrm{A}^{\sim}, \mathrm{B}^{\sim}\right)
$$

As before, we explore the possibility of self-similar quasistationarity in the reaction front: $\mathrm{A}^{\sim}(\eta, t) \sim \mathrm{A}(\eta)$ and $\tilde{B}^{\sim}(\eta, t) \sim$ $\mathrm{B}(\eta)$ as $t \rightarrow \infty$ with $|\eta|<\infty$ fixed. The consequence of the quasistationarity assumption in eq 31 is

$$
\mathrm{A}^{\prime \prime}(\eta)=\lim _{t \rightarrow \infty} t^{\gamma} r\left(t^{-\gamma} \mathrm{A}(\eta), \mathrm{B}(\eta)\right) \quad(|\eta|<\infty)
$$

Since $A^{\prime \prime}(\eta)=0$ cannot satisfy the boundary condition A $(-\infty)=0$ (except in the trivial case $A(\eta)=0)$, the limit on the right-hand side of eq 32 must be nonzero (and finite), which is possible only if $r(a, b)$ is linear in $a$, i.e.

$$
r(a, b)=a \cdot f(b)
$$

for some function $f(b)$. Therefore, the experimental facts $w(t)$ $\sim t^{0}$ and $x_{\mathrm{f}}(t) \sim t^{1 / 2}$ are consistent with the one-dimensional, mean-field model only if the reaction rate is first order in the diffusing species.

Next we make the same transformation in eq 11 and replace the reaction term with eq 33 to obtain

$$
\frac{\partial \mathrm{B}^{\tilde{2}}}{\partial t}+v t^{-1 / 2} \frac{\partial \mathrm{B}^{\tilde{2}}}{\partial \eta}=-q t^{-\gamma} \mathrm{A} \tilde{f}\left(\mathrm{~B}^{\tilde{}}\right)
$$

By inspection, quasistationarity is possible only if $\gamma=1 / 2$, which would imply $r(a, b) \sim t^{-1 / 2} \mathrm{~A}(\eta) f(\mathrm{~B}(\eta))$. Therefore, we conclude $\beta=1 / 2$ once again, and the physical argument given in the previous section is found to have sound mathematical justification.

With these results we arrive at a third-order system of nonlinear ordinary differential equations for the concentration fields in the reaction front,

$$
\begin{gathered}
\mathrm{A}{ }^{\prime \prime}-\mathrm{A} \cdot f(\mathrm{~B})=0 \\
v \mathrm{~B}^{\prime}+q \mathrm{~A} \cdot f(\mathrm{~B})=0
\end{gathered}
$$

These equations may be combined to eliminate the reaction term and integrated once using the boundary conditions ahead of the front, $\mathrm{A}(-\infty)=0$ and $\mathrm{B}(-\infty)=1$ to obtain

$$
q \mathrm{~A}^{\prime}=v(1-\mathrm{B})
$$

Before proceeding with another integration, however, a third

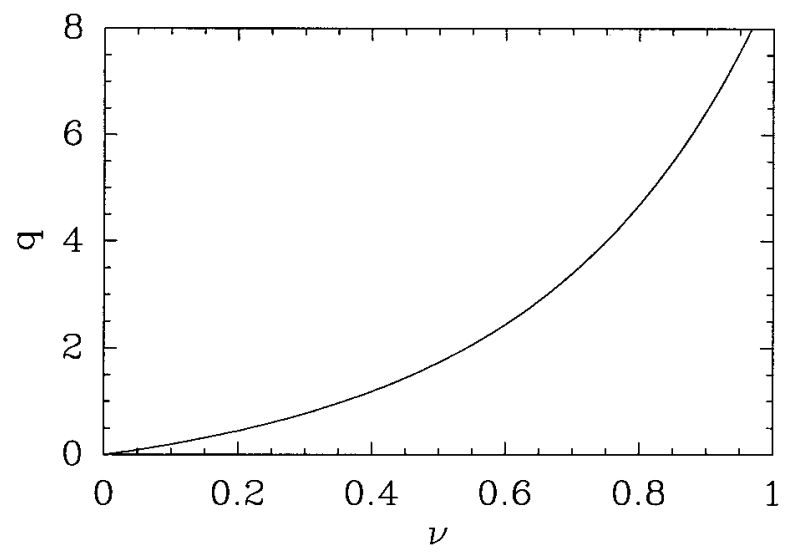

Figure 8. Exact asymptotic dependence of $v$, the square root of the dimensionless diffusion constant of the reaction front, on the asymmetry parameter $q$, predicted by eq 39 .

boundary condition is needed, which comes from asymptotic matching with the diffusion layer.

D. Asymptotic Matching. In mathematical terms, our equations possess an "internal boundary layer". ${ }^{60}$ The reaction front, defined by $|x+2 v \sqrt{t}|=O(1)$, acts as the "inner region", while the diffusion layer, defined by $\sqrt{t}=O(|x+2 v \sqrt{t}|)$, acts as the "outer region". For consistency, the "inner limit" $(\zeta \rightarrow$ 0 ) of the outer approximation, eq 18, must match the "outer limit" $(\eta \rightarrow \infty)$ of the inner approximation, eq 29 . We have shown that $\gamma>0$ is required to describe the experimental data, which means that $a(x, t)$ approaches zero uniformly in the reaction front. Therefore, by matching at zeroth order, we obtain $A(0)=A_{\mathrm{o}}=0$, but this does not provide the missing boundary condition for the reaction front. At the next (linear) order we have, as $t \rightarrow \infty$,

$$
\frac{\partial a}{\partial x}= \begin{cases}\frac{\partial A}{\partial \zeta} \frac{\partial \zeta}{\partial x} \sim \frac{A^{\prime}(\zeta)}{2 \sqrt{t}} & 0<\zeta<\infty \\ \frac{1}{\sqrt{t}} \frac{\partial A}{\partial \eta} \frac{\partial \eta}{\partial x} \sim \frac{A^{\prime}(\eta)}{\sqrt{t}} & |\eta|<\infty\end{cases}
$$

and by matching, we conclude $\mathrm{A}^{\prime}(\infty)=\mathrm{A}_{1}$, where $\mathrm{A}_{1}(v) \equiv$ $A^{\prime}(0) / 2$ can be expressed in terms of $v(q)$ using eq 27. In light of eq 24 , the matching condition for $b(x, t)$ is $\mathrm{B}(\infty)=0$.

The matching conditions allow us to derive an exact expression for $v(q)$ and hence the asymptotic front position $x_{\mathrm{f}}(t)=$ $2 v \sqrt{t}$. Taking the limit $\eta \rightarrow \infty$ in eq 37 using $\mathrm{A}^{\prime}(\infty)=\mathrm{A}_{1}$ and $\mathrm{B}(\infty)=0$, we obtain $q \mathrm{~A}_{1}(v)=v$. By substituting $\mathrm{A}_{1}(v)$ from eq 27, we obtain the desired expression for $v(q)$,

$$
v=F^{-1}(q) \quad \text { where } \quad F(x) \equiv \sqrt{\pi} x \mathrm{e}^{x^{2}}[1+\operatorname{erf}(x)]
$$

which has also been derived by Koza. ${ }^{31}$ The relation $q=F(v)$ is plotted in Figure 8 and will be used in the next section to estimate $q$ from the experimentally measured value of $\nu$.

With these results, we are led to a second-order, nonlinearboundary-value problem for the reaction front concentration of the diffusing species:

$$
\mathrm{A}^{\prime \prime}=\mathrm{A} \cdot f\left(1-\mathrm{A}^{\prime} / \mathrm{A}_{1}\right), \quad \mathrm{A}(-\infty)=0, \quad \mathrm{~A}^{\prime}(\infty)=\mathrm{A}_{1}
$$

Note that eq 40 is invariant to translation $\eta \rightarrow \eta+\eta_{\mathrm{o}}$, where $\eta_{\mathrm{o}}$ is an undetermined constant depending on the initial conditions that precisely defines the location of the reaction front (e.g., as the point of maximal reaction rate). 
Since it is difficult to accurately measure the reaction-front concentration fields in our experiments, we stop here and refer the reader to the article of Bazant and Stone ${ }^{10}$ for the integration of this boundary-value problem and other analytical results in the case $f(b)=b^{m}, m \geq 1$.

\section{Experimental Test of the Theoretical Model}

A. Check of the Exact Asymptotic Predictions. In section III we showed that as corrosion proceeds, the reaction front moves with the time as $X_{\mathrm{f}}(T) \sim T^{1 / 2}$ and does not spread $(w(T)$ $\sim T^{\alpha}$ with $\alpha=0$ ) and the width $W_{\mathrm{d}}$ of the depletion layer increases with the time as $W_{\mathrm{d}}(T) \sim T^{1 / 2}$. In section IV we showed that these observations are consistent with the predictions of a one-dimensional A $+\mathrm{B}$ (static) $\rightarrow \mathrm{C}$ (inert) mean-field model with a reaction rate that is first order in the diffusing species A. By solving the mean-field equations, we derived not only the scaling exponents for $X_{\mathrm{f}}(T)$ and $W(T)$ but also the prefactors and the exact asymptotic shape of the concentration profile of the diffusing reactant as a function of the reduced coordinate $\zeta$ $=\left[X-X_{\mathrm{f}}(T)\right] / 2 \sqrt{D T}$. In this section, we quantitatively test these theoretical predictions against the experimental results.

1. Movement of the Front. In dimensional units eq 19 reads

$$
-X_{\mathrm{f}}=2 v(q) \sqrt{D T}
$$

Therefore, from a $\log -\log$ plot of $X_{\mathrm{f}}$ as a function of $T$ one gets the value of $v$, and $q$ can then be deduced from eq 39. In our experimental system, $q$ is linearly related to a characteristic property of the electrolyte, namely the transference number of the cation, through $q=1-t_{+}$. To derive the values of $q$ and $t_{+}$from eq 41 , we need an accurate value of the diffusion coefficient of the electrolyte. $D$ is likely to depend on the concentration of $\mathrm{CuCl}_{2}$, but to our knowledge, has not been tabulated for $\mathrm{CuCl}_{2}$. Hereafter, we use the value $D=(1.0 \pm$ $0.1) \times 10^{-5} \mathrm{~cm}^{2} \cdot \mathrm{s}^{-1}$, determined independently by our interferometric technique.

The two sets of experimental data in of Figure 3 give $2 v \sqrt{D}$ $=(1.7 \pm 0.1) \times 10^{-3} \mathrm{~cm} \cdot \mathrm{s}^{-1 / 2}$, therefore $v=0.27 \pm 0.02$ and $t_{+}=0.33 \pm 0.05$ from eq $39, q=F(v)$. Note that $t_{+} \approx 0.3$ (for a $0.5 \mathrm{~mol} \cdot \mathrm{L}^{-1}$ electrolyte) is quite consistent with the corresponding value at infinite dilution $t_{+}^{\infty}=0.4$, since $t_{+}$is likely to be a decreasing function of the concentration. ${ }^{49}$ Although we have not directly measured the transference number $t^{+}$of the $\mathrm{Cu}^{2+}$ cation, its reasonable value just inferred from the observed front speed via eq 39 constitutes a successful prediction of the one-dimensional mean-field model.

2. Width of the Depletion Zone and Whole Concentration Profile. In this section, we analyze the experiments performed with a higher electrolyte concentration, namely $1.0 \mathrm{~mol} \cdot \mathrm{L}^{-1}$ $\mathrm{CuCl}_{2}$. The concentration profile in the laboratory frame can be written in dimensional units using eq 26 and the definition of $\zeta$ :

$$
a(X, T)=\frac{\operatorname{erf}(X / 2 \sqrt{D T})+\operatorname{erf}(v)}{1+\operatorname{erf}(v)}
$$

Note that $a(X, T)$ is used in the experimental parts to denote $\rho_{\mathrm{A}}(X, T) / \rho_{\mathrm{A}}^{\circ}$. A characteristic feature of these profiles (and the experimental data in Figure 5) is that they exhibit a fixed point with ordinate

$$
a(X=0, T)=\frac{\operatorname{erf}(v)}{1+\operatorname{erf}(v)}
$$

Since $a(0, T)$ depends only on $q$, a value of $q$ can be deduced from Figure 5, which shows the concentration profiles during the corrosion of a copper deposit obtained by electrodeposition from a $1.0 \mathrm{~mol} \cdot \mathrm{L}^{-1} \mathrm{CuCl}_{2}$ solution. We find $a(X=0, T)=0.25$ \pm 0.01 , which implies $v=0.30 \pm 0.01$. From eq 39 the meanfield model would predict $q=0.79 \pm 0.06$. As expected, the inferred value of the transference number, $t_{+}=1-q=0.21$ \pm 0.06 , for this $1.0 \mathrm{~mol} \cdot \mathrm{L}^{-1} \mathrm{CuCl}_{2}$ solution is lower than the value of $0.33 \pm 0.05$ at $0.5 \mathrm{~mol} \cdot \mathrm{L}^{-1}$ computed above. This value is somewhat smaller than expected on the basis of concentration effects (see below). Note that we have not directly measured the ratio $q=\rho_{\mathrm{A}}^{\circ} / \rho_{\mathrm{B}}^{\circ}$ or the transference number $t^{+}$in the experiments described in this paper, but the value of $q=0.79$ just obtained from eq 43 is necessary for comparison with the mean-field model (without any other adjustable parameters). Therefore, we will use $q=0.79$ in the following analysis of the experimental runs in $1 \mathrm{~mol} \cdot \mathrm{L}^{-1} \mathrm{CuCl}_{2}$ electrolyte.

From eq 26 the width $W_{\mathrm{d}}$ of the diffusion layer (with dimensions) is given by

$$
W_{\mathrm{d}}(T)=\left(\left.\partial_{\mathrm{X}} a(X, T)\right|_{X=X_{\mathrm{f}}}\right)^{-1}=\left(\frac{\exp \left(-v^{2}\right)}{\sqrt{\pi D T}(1+\operatorname{erf}(v))}\right)^{-1}
$$

From an experimental point of view, it is simpler to measure $a(X, T)$ at $X=0$ rather than at $X=X_{\mathrm{f}}(T)$, so we consider the temporal evolution of the gradient of $a(X, T)$ at $X=0$. From eq 42 we obtain

$$
\left.\frac{\partial a(X, T)}{\partial X}\right|_{X=0}=\frac{1}{\sqrt{\pi D T}(1+\operatorname{erf}(v))}
$$

and $W_{\mathrm{d}}(T)=\exp \left(v^{2}\right) /\left.\partial_{X} a(X, T)\right|_{X=0}$. Figure 6 shows the quantitative agreement between the experimental values of $\left.\partial_{X} a\right|_{X=0}$ and the function of eq 45 plotted for $D=10^{-5} \mathrm{~cm}^{2} \cdot \mathrm{s}^{-1}$ and $q=$ 0.79 . Note that $D$ and $q$ are deduced from previous analysis and are not adjustable parameters.

Continuing our quantitative analysis of the experimental concentration field, we plot in Figure 9 the asymptotic shape of the concentration profile. To determine $a(\xi)$ from $a(X, T)$, we compute $\xi$ using $\zeta=(X / 2 \sqrt{D T})+v(q)$, with $q=0.79$ and $D=10^{-5} \mathrm{~cm}^{2} \cdot \mathrm{s}^{-1}$ and adjust the origin of the abscissa to the initial front of copper position, to ensure that $\tilde{A}(\xi=0, T)=0$ for all $T$. For comparison we also show in the same plot the theoretically predicted $A(\xi)$ function computed from eqs 26 and 39 with $q=0.79$.

To focus on the region of the reaction front, the experimental data are plotted in Figure 10 according to the linearized version of eq 26

$$
a \sqrt{D T}=A^{\prime}(0) \frac{X-X_{\mathrm{f}}}{2}=\frac{2 \mathrm{e}^{-v^{2}}}{\sqrt{\pi}(1+\operatorname{erf}(v))} \frac{X-X_{\mathrm{f}}}{2}
$$

Since $\left(X-X_{\mathrm{f}}\right) / 2$ is proportional to the reaction-front similarity variable $\eta$ in eq 30 , the mean-field model would predict a collapse of this data to a single curve given by the solution of eq 40 .

Unfortunately, the noise in the experimental data washes out the exact concentration profiles in the reaction front on this scale, but it is clear that the width of the reaction front has the asymptotic scaling $w \sim t^{\alpha}$ with $\alpha=0$. Moreover, the asymptotic shape of the concentration distribution is quite consistent with the solutions to eq 40 given in ref 10 . Note that the decay of slope of the reaction-front concentration $A^{\prime}(\eta)$ toward its limiting value in the diffusion layer $A_{1}$ in Figure 10 appears to 


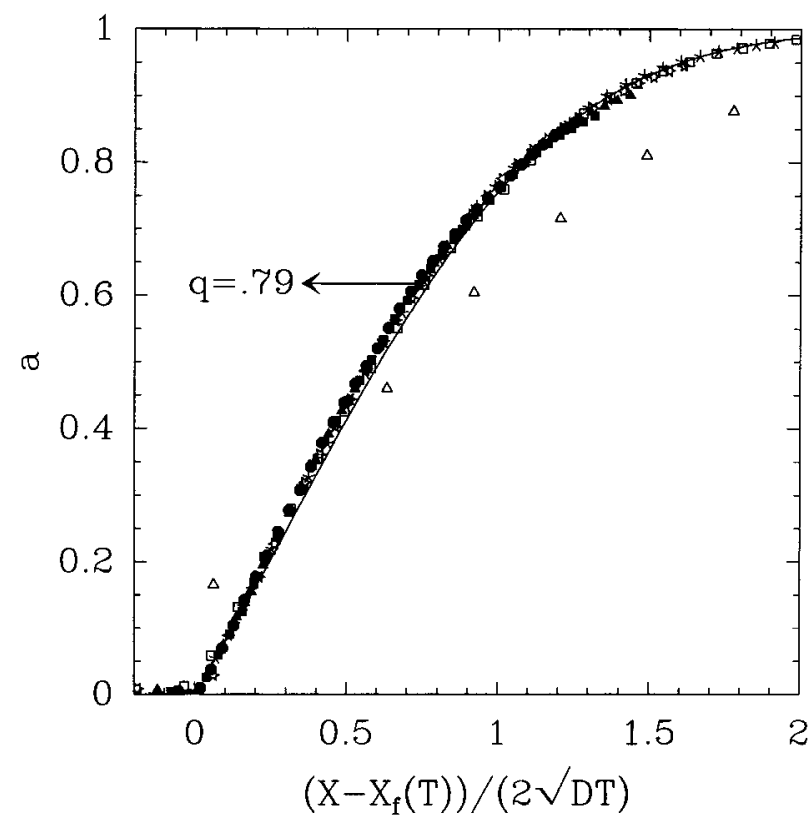

Figure 9. Collapse of the experimental concentration data in the diffusion layer plotted versus the similarity variable $\left(X-X_{\mathrm{f}}\right) / 2 \sqrt{D T}$ compared with the theoretically predicted asymptotic experimental similarity function $A(\xi)$ (the solid line). The profiles are the same as those plotted in Figure 5, but only one point out of 20 is shown on this plot for clarity.

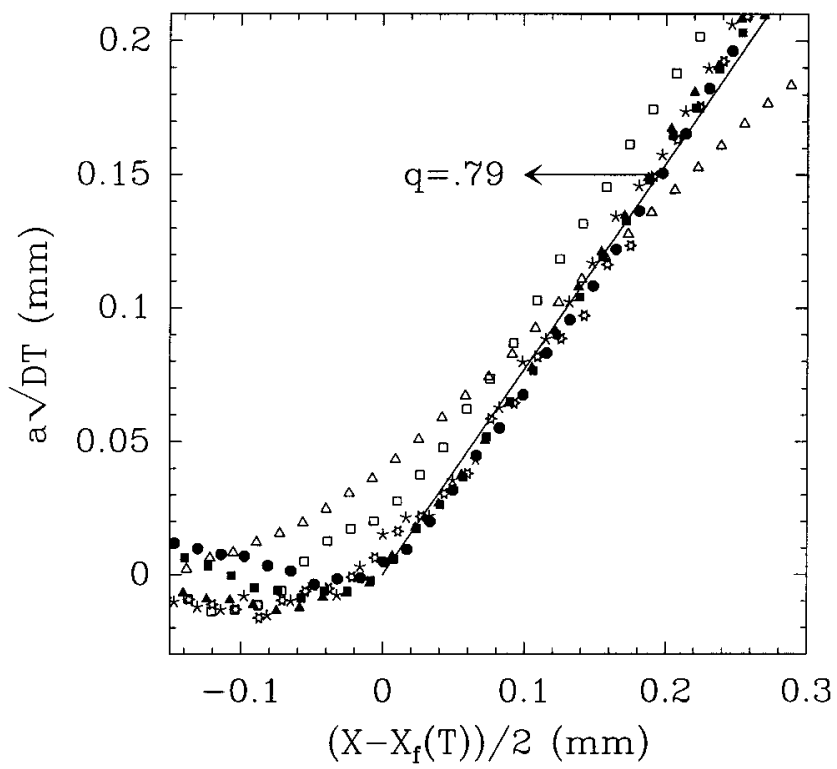

Figure 10. Collapse of the experimental concentration data in the reaction front plotted versus the similarity variable $\left(X-X_{\mathrm{f}}\right) / 2$. The solid line shows the linearized extension of the similarity function $A(\zeta)$ from the diffusion layer (see Figure 9) extended into the reaction front. These profiles are the same as those plotted in Figure 5, but only one point out of 4 is shown on this plot for clarity. The negative concentration values are artifacts of the interferometric technique and have no physical meaning.

be quite fast. If this decay were exponential rather than a (much slower) power law, then according to the mean-field model ${ }^{10}$ the reaction rate would have to be first order in the static reactant $m=1$, i.e., $f(b)=b$ or $r(a, b)=a b$, but it is impossible to reach this conclusion definitively from our data.

As shown in Figures 9 and 10, all of the measured concentration profiles collapse to the single asymptotic curve predicted for $q=0.79$ over the whole length scales investigated in the experiment. This quantitative agreement between the experi- mental and theoretical concentration profiles of the diffusing reactant independent of the length scale strongly support our modeling of this corrosion experiment with a one-dimensional $\mathrm{A}+\mathrm{B}$ (static) $\rightarrow \mathrm{C}$ (inert) mean-field model.

B. The Transient. The A $+\mathrm{B} \rightarrow \mathrm{C}$ mean-field model with two diffusing reactants exhibits many surprising and nontrivial behaviors at short times (see refs 30 and references therein, 26, and 61). In this case, some microscopic parameters like the reaction constant(s) can be determined from these short time behaviors. In particular, at a time inversely proportional to the microscopic reaction constant, the global reaction rate switches from an initial $t^{1 / 2}$ increase to a subsequent $t^{-1 / 2}$ decrease. ${ }^{30}$ Moreover, in the reversible $\mathrm{A}+\mathrm{B} \rightleftharpoons \mathrm{C}$ system, a crossover between irreversible and reversible regimes can be observed at long times ${ }^{61}$ and the value of the backward reaction constant can be inferred from the crossover time. ${ }^{26}$

In the present case of one static reactant, it is also possible to express the transient decay to the asymptotic solution in terms of the reaction orders $m$ and $n$ for the one-dimensional meanfield model. ${ }^{10}$ In our experiment, however, the transient behavior is determined by a superposition of different mechanisms since our system is not really one-dimensional or homogeneous. We now show that the transient behavior appears to be governed by two-dimensional geometric effects that hide the kinetic features by analyzing the detail of the experimental runs.

Looking at Figure 4a, note that the concentration field is not one-dimensional at the early stages of the corrosion experiment: the isoconcentration lines closely follow the jagged outline of the deposit in the region near the tips. The amplitude $G$ of the modulation of the leftmost isoconcentration line (the closest to the copper cluster) is about $0.4 \mathrm{~mm}$. This system clearly cannot be viewed as one-dimensional until the front has traveled at least a distance on the order of $G$. In Figure 3, note that the time of the transient regime (before the asymptotic $t^{1 / 2}$ behavior sets in) closely corresponds to the time needed for the front to move across a distance $G \sim 0.4 \mathrm{~mm}$. (This twodimensional geometric effect also may explain why the initial movement of the front is slower than the asymptotic behavior, as shown in Figure 3.)

To further support this hypothesis, we now study the relaxation dynamics of the concentration field. In Figure 11a, is plotted the isoconcentration line corresponding to $a=\rho_{\mathrm{a}} / \rho_{\mathrm{a}}^{\circ}$ $=0.1$, just after the current has been switched off. This line is not continuous, because the concentration field cannot be extracted by interferometry in the zones containing the deposit. This line defines a function $X(Y)$, roughly periodic, of amplitude $G(T)$ and period $\lambda \sim 1 \mathrm{~mm}$. It is reasonable to expect that the characteristic time for the relaxation of this modulation of the concentration field toward a flat two-dimensional profile is the time $\tau_{\mathrm{f}}$ needed for the front of copper to move from its starting position $\left(X_{\mathrm{f}}(T=0)\right)$ on the length scale $G(T=0)=0.3 \mathrm{~mm}=$ $2 v \sqrt{D \tau_{\mathrm{f}}}$, which yields the estimate $\tau_{\mathrm{f}}=G(0)^{2} / 4 v^{2} D \sim 250 \mathrm{~s}$. Moreover, in light of the analysis of Krug ${ }^{62}$ described below, it is also reasonable to expect that the functional form of the decay will be exponential.

In Figure 11b, we plot $\log (G(T) / G(0))$ as a function of the dimensionless time $T / \tau_{\mathrm{f}}$. The relaxation is well fitted by an exponential function, with a characteristic time close to $\tau_{\mathrm{f}}$, which supports our hypothesis. Therefore in our experiments, the transient behavior is directly linked to the relaxation of the initial two-dimensional concentration field toward a Y-invariant profile and cannot provide information on the kinetics independently.

C. Physical Relevance of the One-Dimensional Mean-Field Model. In the previous sections, we have demonstrated the 

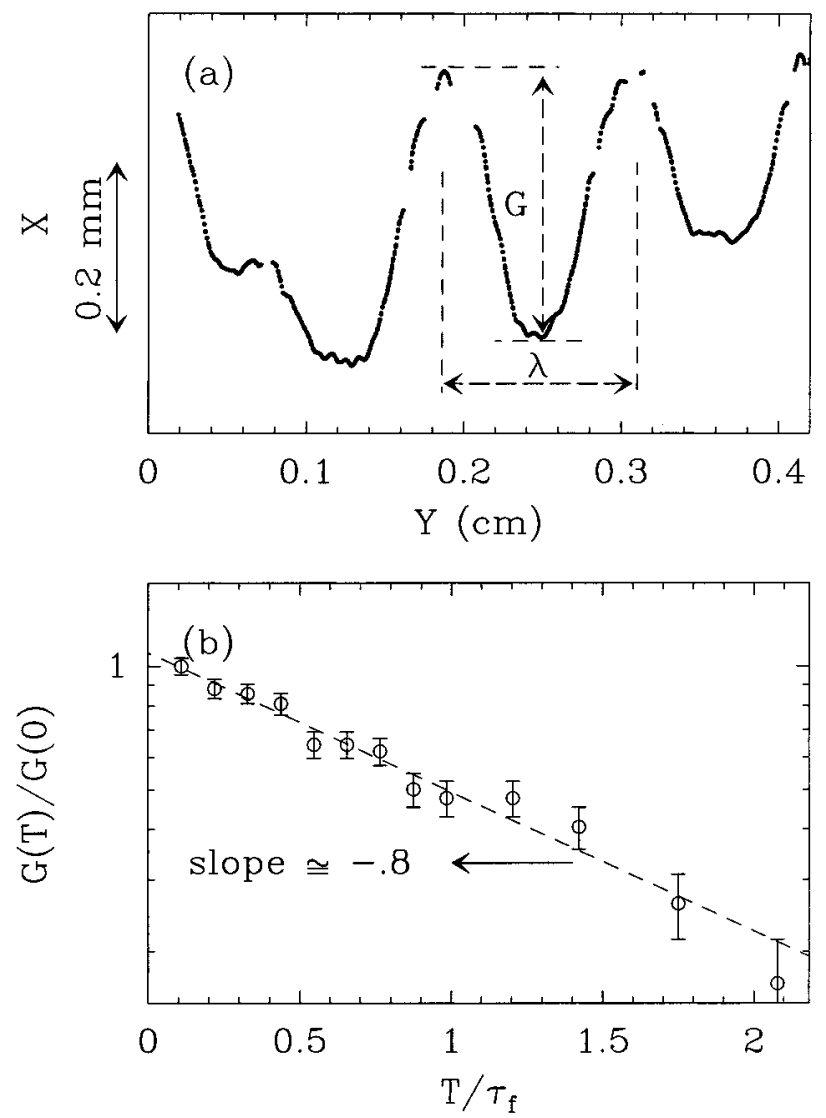

Figure 11. Relaxation of the two-dimensional initial concentration field at the beginning of the dissolution. (a) Isoconcentration line $a=0.1$, for $T=27 \mathrm{~s}$. The deposit has been grown from a $1 \mathrm{~mol} \cdot \mathrm{L}^{-1} \mathrm{CuCl}_{2}$ solution, at $j=68 \mathrm{~mA} \cdot \mathrm{cm}^{-2}$ over $15 \mathrm{~min}$. (b) log-linear plot of the evolution of the amplitude $G$ of the modulation of $A$ concentration, as shown in (a), versus the reduced time $T / \tau_{\mathrm{f}}=4 v^{2} D T / G(0)^{2}$.

quantitative agreement between the behavior of our thin gap corrosion system and various predictions of a one-dimensional mean-field model. This agreement is not obvious a priori, and therefore we close in this section by giving physical arguments to explain this surprising fact.

1. No Inhibition of Diffusion or Reaction by $\mathrm{CuCl}$. In Figure 4 we see that the product of the reaction does not seem to disturb the concentration field of the diffusing reactant $\mathrm{A}$. To understand this fact, we consider the volume occupied by the product $\mathrm{CuCl}$ in the cell. We know from eq 7 that the mean concentration of copper before the dissolution is $\approx 2 \rho_{\mathrm{A}}^{\circ}$. We deduce from eq 4 that if $\mathrm{CuCl}$ does not diffuse (which is verified in our experiments), the mean concentration of $\mathrm{CuCl}$ is twice the initial concentration of copper, which is approximately 4 times the initial concentration of $\mathrm{CuCl}_{2}$ in the bulk, i.e., $2 \mathrm{~mol} \cdot \mathrm{L}^{-1}$. Since the density and molecular weight of $\mathrm{CuCl}$ are $3.38 \mathrm{~g} \cdot \mathrm{cm}^{-3}$ and $99 \mathrm{~g} \cdot \mathrm{mol}^{-1}$, respectively, the volume occupied by the solid $\mathrm{CuCl}$ after the dissolution is roughly $5 \%$ of the total local volume. Therefore, the small crystals of $\mathrm{CuCl}$ do not significantly alter the volume free for the diffusion of $\mathrm{CuCl}_{2}$. Moreover, because the $\mathrm{CuCl}$ crystallites do not adhere to the copper metal branches and fall to the bottom of the thin gap cell, the surface of the copper cluster is constantly renewed and "ready" for corrosion by $\mathrm{CuCl}_{2}$.

2. Stable Front, Asymptotically One-Dimensional. The fact that the dissolution process builds a stable (flat) interface can be understood by considering that diffusion-limited corrosion is the "time-reversed" process of diffusion-limited aggregation and that the fluctuations of the interface decay rather than grow to reach a stable flat front asymptotically. Krug62 showed that periodic perturbations of a flat front of wavelength $\lambda$ in the direction perpendicular to the direction of motion of the interface would decay with a characteristic time $\tau=\lambda / v$. The stability of the corrosion front can therefore be qualitatively understood with the following argument: the electrolyte most easily reaches the most exposed or least screened parts of the copper deposit. These bulges are dissolved first, and the interface is smoothed.

3. Relevance of 1D Approximation of the Concentration Field. In the long-time asymptotic regime, the modulation of the initial concentration of reactant $\mathrm{A}\left(\mathrm{CuCl}_{2}\right)$ relaxes toward a flat concentration profile along the direction $Y$ whose shape is given by eq 26 . However, the concentration of the static reactant $\mathrm{B}$ $(\mathrm{Cu})$, as well as the concentration of the product $\mathrm{C}(\mathrm{CuCl})$ keep a periodic shape along the $Y$ direction, which somehow does not alter the one-dimensional asymptotic solution. The largest characteristic length of the deposit in the direction parallel to the front $(Y)$ is the mean distance $\lambda$ between the trees. This puzzling observation can be understood by comparing the relaxation time of the perturbations of $\rho_{\mathrm{A}}(X, Y)$ along $Y, \tau_{\mathrm{d}} \sim$ $\lambda^{2} / D$, with the time needed by the front of copper to move on the same length, $\tau_{\mathrm{f}} \sim \lambda / \dot{X}_{\mathrm{f}}=\lambda \sqrt{T} / \nu \sqrt{D}$. Since $\tau_{\mathrm{f}}$ increases with time $T$, in the asymptotic regime it will be much greater than $\tau_{\mathrm{d}}$. Therefore, whereas $\rho_{\mathrm{B}}$ is highly correlated along the $Y$ direction due to the structure of the solid deposit, there are eventually no fluctuations in $\rho_{\mathrm{A}}$ along this direction.

4. Departure from Pure Diffusion in the Reaction Zone. The fact that the transference number $t^{+}$deduced from $t^{+}=1-q$ and the inferred value of $q=F(v)$ from eq 39 decreases significantly from 0.33 to 0.21 when the concentration of $\mathrm{CuCl}_{2}$ is increased from 0.5 to $1 \mathrm{~mol} \cdot \mathrm{L}^{-1}$ is unlikely to be caused solely by a pure salt-concentration effect. It is also possible that convection produced by the sedimentation of $\mathrm{CuCl}$ crystallites toward the bottom of the cell could artificially increase the effective diffusion coefficient close to the reaction front by convective mixing. This would cause an increase of $v(q)$ (the prefactor for the speed of the reaction front), which could at least partly explain the difference in the inferred $q$ values, and therefore also in the effective $t^{+}$values.

\section{Conclusion}

We have shown that after long times the corrosion of highly porous copper clusters can be understood as a one-dimensional, homogeneous, mean-field $\mathrm{A}+\mathrm{B} \rightarrow \mathrm{C}$ reaction-diffusion process with one diffusing and one static reactant. This is the first experimental analysis of such a situation where only one reactant is free to diffuse through the other one. Whereas one would expect highly complex dynamics and a possible breakdown of the mean-field approximation when the reaction is confined to a porous (fractal) interface, we show that in this particular corrosion system, the dynamics are equivalent to those expected for a homogeneous system. The strength of our demonstration is built on precise measurements of the concentration field of the diffusing species by interferometry, which are compared quantitatively with analytical predictions of the one-dimensional mean-field model.

Acknowledgment. We are very grateful to Y. Sorin and G. Gadret for their technical assistance with the optical setup. We also thank H. A. Stone, E. Clément, J. Elezgaray, and A. Arneodo for stimulating and fruitful discussions. This work was supported (mainly) by the Centre National d'Etudes Spatiales under Grant No. 97/CNES/071/6850 and also (partially) by an NFS infrastructure grant to the MIT Department of Mathematics. 


\section{References and Notes}

(1) Ben-Jacob, E. Contemp. Phys. 1993, 34, 247 and references therein.

(2) Epstein, I.; Showalter, K. J. Phys. Chem. 1996, 100, 13132 and references therein.

(3) Ben-Jacob, E. Contemp. Phys. 1997, 38, 205.

(4) Smoluchowski, M. V. Phys. Z. 1916, 17, 557.

(5) Collins, F.; Kimball, G. J. Colloid Sci. 1949, 4, 425.

(6) Calef, D.; Deutch, J. Annu. Rev. Phys. Chem. 1983, 34, 493.

(7) Galfi, L.; Racz, Z. Phys. Rev. Lett. 1988, 38, 3151.

(8) Schenkel, A.; Wittwer, P.; Stubbe, J. Physica D 1993, 69, 135.

(9) Koza, Z. J. Stat. Phys. 1996, 85, 179.

(10) Bazant, M. Z.; Stone, H. A. Sumitted to Physica D (http:// xxx.lanl.gov/abs/physics/9904008).

(11) Jiang, Z.; Ebner, C. Phys. Rev. A 1990, 42, 7483.

(12) Choppard, B.; Droz, M. Europhys. Lett. 1991, 15, 459.

(13) Cornell, S.; Droz, M.; Chopard, B. Phys. Rev. A 1991, 44, 4826.

(14) Cornell, S.; Droz, M.; Chopard, B. Physica A 1992, 188, 322.

(15) Cornell, S.; Droz, M. Phys. Rev. Lett. 1993, 70, 3824.

(16) Cornell, S.; Koza, Z.; Droz, M. Phys. Rev. E 1995, 52, 3500

(17) Cornell, S.; Droz, M. Physica D 1997, 103, 348.

(18) Araujo, M.; Larralde, H.; Havlin, S.; Stanley, H. Physica A 1992, 191, 168.

(19) Araujo, M.; Larralde, H.; Havlin, S.; Stanley, H. Phys. Rev. Lett. 1993, 71, 3592.

(20) Koza, Z.; Taitelbaum, H. Phys. Rev. E (R) 1996, 54, 1040.

(21) Taitelbaum, H.; Yen, A.; Kopelman, R.; Havlin, S.; Weiss, G. H. Phys. Rev. E 1996, 54, 5942. 187.

(22) Koo, Y.; Li, L.; Kopelman, R. Mol. Cryst. Liq. Cryst. 1990, 183,

(23) Koo, Y. L.; Kopelman, R. J. Stat. Phys. 1991, 65, 893.

(24) Taitelbaum, H.; Koo, Y. L.; Havlin, S.; Kopelman, R.; Weiss, G. Phys. Rev. A 1992, 46, 2151.

(25) Taitelbaum, H.; Vilensky, B.; Lin, A.; Koo, Y.-E. L.; Kopelman, R. Phys. Rev. Lett. 1996, 77, 1640.

(26) Yen, A.; Koo, Y.-E. L.; Kopelman, R. Phys. Rev. E 1996, 54, 2447. (27) Yen, A.; Lin, A.; Koo, Y.-E. L.; Vilensky, B.; Taitelbaum, H.; R., Kopelman, J. Phys. Chem. A 1997, 101, 2819.

(28) Yen, A.; Kopelman, R. Phys. Rev. E 1997, 56, 3694

(29) Yen, A.; Shi, Z.-Y.; Kopelman, R. Phys. Rev. E 1998, 57, 2438.

(30) Taitelbaum, H.; Koza, Z. Philos. Mag. B 1998, 77, 1389.

(31) Koza, Z. Physica A 1997, 240, 622.

(32) Havlin, S.; Araujo, M.; Lereah, Y.; Larralde, H.; Shehter, A.; Stanley, H.; Trunfio, P.; Vilensky, B. Physica A 1995, 221, 1.

(33) Data Translation frame grabber $(768 \times 512)$ and public domain $\mathrm{NIH}$ Image program, developed at the U.S. National Institutes of Health and available on the Internet at http://rsb.info.nih.gov/nih-image/.

(34) Léger, C.; Elezgaray, J.; Argoul, F. Phys. Rev. Lett. 1997, 78, 5010.

(35) Crennell, K.; Gasvik, K.; Yatagai, T.; Creath, K.; Kujawinska, M.;

Robinson, D.; Halliwell, N.; Pickering, C.; Hariharan, P. In Interferogram analysis; Robinson, D., Reid, G., Eds.; Institute of Physics Publishing: Techno House, Redcliffe Way, Bristol, BS1 6NX, England, 1993.

(36) Léger, C.; Elezgaray, J.; Argoul, F. Phys. Rev. E 1998, 58, 7700.

(37) Oberholtzer, F.; Barkey, D.; Wu, Q. Phys. Rev. E 1998, 57, 6955.

(38) Trigueros, P.; Sagues, F.; Claret, J. Phys. Rev. E 1994, 49, 4328.

(39) Lòpez-Salvans, M.-Q.; Trigueros, P.; Vallmitjana, S.; Claret, J.;

Sagues, F. Phys. Rev. Lett. 1996, 76, 4062.

(40) Texier, F.; Servant, L.; Bruneel, J.; Argoul, F. J. Electroanal. Chem. 1998, 446, 189.

(41) Newman, J. Electrochemical Systems; Prentice Hall: Englewood Cliff, NJ, 1991.

(42) Parsons, R. Chem. Rev. 1980, 90, 813.

(43) Bard, A.; Faulkner, L. Electrochemical methods. Fundamentals and applications; John Wiley and Sons: New York, 1980.

(44) Newman, J. Trans. Faraday Soc. 1965, 61, 2229.

(45) Chazalviel, J.-N. Phys. Rev. A 1990, 42, 7355. 2016.

(46) Elezgaray, J.; Léger, C.; Argoul, F. J. Electrochem. Soc. 1998, 145 ,

(47) Melrose, J. Phys. Rev. Lett. 1990, 65, 3009.

(48) Fleury, V.; Chazalviel, J.-N.; Rosso, M.; Sapoval, B. J. Electroanal. Chem. 1990, 290, 249.

(49) Fleury, V.; Rosso, M.; Chazalviel, J.-N.; Sapoval, B. Phys. Rev. A 1991, 44, 6693.

(50) Bazant, M. Z. Phys. Rev. E 1995, 52, 1903.

(51) Kuhn, A.; Argoul, F.; Muzy, J.; Arneodo, A. Phys. Rev. Lett. 1994, $73,2998$.

(52) Kuhn, A.; Argoul, F. J. Electroanal. Chem. 1995, 397, 93. 75

(54) Arneodo, A.; Argoul, F.; Bacry, E.; Elezgaray, J.; Muzy, J. F. Ondelettes, multifractales et turbulences: de l'ADN aux croissances cristallines; Diderot éditeur, Arts et Sciences, Paris, 1995.

(55) Barenblatt, G. I. Similarity, Self-Similarity and Intermediate Asymptotics; Cambridge University Press: Oxford, U.K., 1979.

(56) Huth, J.; Swinney, H.; McCormick, W.; Kuhn, A.; Argoul, F. Phys. Rev. E 1995, 51, 3444

(57) Chazalviel, J.-N.; Rosso, M.; Chassaing, E.; Fleury, V. J. Electroanal. Chem. 1996, 407, 61.

(58) Ben-Naim, E.; Redner, S. J. Phys. A. Math. Gen. (L) 1992, 25, 575 .

(59) Abramowitz, M.; Stegun, I. A. Handbook of Mathematical Functions; Dover: New York, 1965.

(60) Bender, C.; Orszag, S. Advanced Mathematical Methods for Scientists and Engineers; McGraw-Hill: New York, 1978.

(61) Chopard, B.; Droz, M.; Karapiperis, T.; Racz, Z. Phys. Rev. E (R) $1993,47,40$.

(62) Krug, J.; Meakin, P. Phys. Rev. Lett. 1991, 66, 703. 